\title{
Classification techniques on computerized systems to predict and/or to detect Apnea: A systematic review
}

\author{
Nuno Pombo a,*, Nuno Garcia ${ }^{a}$, Kouamana Bousson ${ }^{\mathrm{b}}$ \\ ${ }^{a}$ Research Units: Instituto de Telecomunicações and ALLab Assisted Living Computing and Telecommunications Laboratory, Department of Informatics, \\ Universidade da Beira Interior, Covilhã, Portugal and Universidade Lusófona de Humanidades e Tecnologias, Lisbon, Portugal \\ ${ }^{\mathrm{b}}$ Research Unit: LAETA/UBI-AEROG, Department of Aerospace Sciences, Universidade da Beira Interior, Covilhã, Portugal
}

\section{A R T I C L E I N F O}

\section{Article history:}

Received 13 June 2016

Revised 28 December 2016

Accepted 3 January 2017

\section{Keywords:}

Sleep apnea

Machine learning

Classification

Threshold-based classification

Systematic review

\begin{abstract}
A B S T R A C T
Background and objective: Sleep apnea syndrome (SAS), which can significantly decrease the quality of life is associated with a major risk factor of health implications such as increased cardiovascular disease, sudden death, depression, irritability, hypertension, and learning difficulties. Thus, it is relevant and timely to present a systematic review describing significant applications in the framework of computational intelligence-based SAS, including its performance, beneficial and challenging effects, and modeling for the decision-making on multiple scenarios.

Methods: This study aims to systematically review the literature on systems for the detection and/or prediction of apnea events using a classification model.

Results: Forty-five included studies revealed a combination of classification techniques for the diagnosis of apnea, such as threshold-based (14.75\%) and machine learning (ML) models (85.25\%). In addition, the ML models, were clustered in a mind map, include neural networks (44.26\%), regression (4.91\%), instance-based (11.47\%), Bayesian algorithms (1.63\%), reinforcement learning (4.91\%), dimensionality reduction (8.19\%), ensemble learning (6.55\%), and decision trees $(3.27 \%)$.

Conclusions: A classification model should provide an auto-adaptive and no external-human action dependency. In addition, the accuracy of the classification models is related with the effective features selection. New high-quality studies based on randomized controlled trials and validation of models using a large and multiple sample of data are recommended.
\end{abstract}

@ 2017 Published by Elsevier Ireland Ltd.
Abbreviations: AI, Apnea Index; AHI, Apnea and Hypopnea Index; AIRS, Artificial Immune Recognition System; ANN, Artificial Neural Network; ANFIS, Adaptive Neuro-Fuzzy Inference System; AUC, Area Under receiver operating characteristic Curve; BHC, Binary Hierarchical Classification; BNN, Bayesian Neural Network; CAS, Central Sleep Apnea; ECOC, Error Correcting Output Code; ECG, Electrocardiogram; EEG, Electroencephalogram; EMG, Electromyography; EOG, electrooculography; FP, False Positive; FN, False Negative; HI, Hypopnea Index; HMM, Hidden Markov Model; KNN, K-Nearest Neighbor; LDA, Linear Discriminant Analysis; LSSVM, Least Squares Support Vector Machine; LR, Logistic Regression; LVQ Learning Vector Quantization; ML, Machine Learning; MLR, Multi-Linear Regression; MSA, Mixed Sleep Apnea; NARX, Nonlinear AutoRegressive network with eXogenous; OSA, Obstructive Sleep Apnea; PNN, Probabilistic Neural Network; NPV, Negative Predictive Value; PPG, Photoplethysmogram; PPV, Positive Predictive Value; PSG, Polysomnogram; RBFNN, Radial Basis Function Neural Network; RCT, Randomized Controlled Trial; RDI, Respiratory Disturbance Index; ROC, receiver operating characteristic; SAS, Sleep Apnea Syndrome; Sp02, Oxygen Saturation; SRN, Simple Recurrent Network; SVM, Support Vector Machine; TP, True Positive; TN, True Negative; VDA, Voice Activity Detection.

* Corresponding author.

E-mail addresses: ngpombo@ubi.pt (N. Pombo), ngarcia@di.ubi.pt (N. Garcia), bousson@ubi.pt (K. Bousson).

\section{Introduction}

Sleep apnea syndrome (SAS) is defined as a temporary closure of the upper airway during sleep when air is prevented from entering lungs which may results on the complete cessation of breathing for more than $10 \mathrm{~s}$ in adults [1]. This is typically, accompanied by a reduction in blood oxygen saturation and leads to arousal from sleep in order to breathe. In addition, repetitive obstructive events during sleep are hypothesized to cause intermittent hypoxia, resulting in activation of oxygen free radicals and an oxidative stress response.

As SAS events are classified according to whether the patient exhibits respiratory effort, then the presence of abdominal and thoracic effort for continuing breathing while air flow completely stops, is called Obstructive Sleep Apnea (OSA) representing the most common pattern of SAS. On the contrary, when a complete cessation of both respiratory movements and airflow during at least $10 \mathrm{~s}$, is considered as Central Sleep Apnea (CSA). Finally, the combination of these two symptoms, defined by a central respiratory pause followed, in a relatively short interval of time, by an 


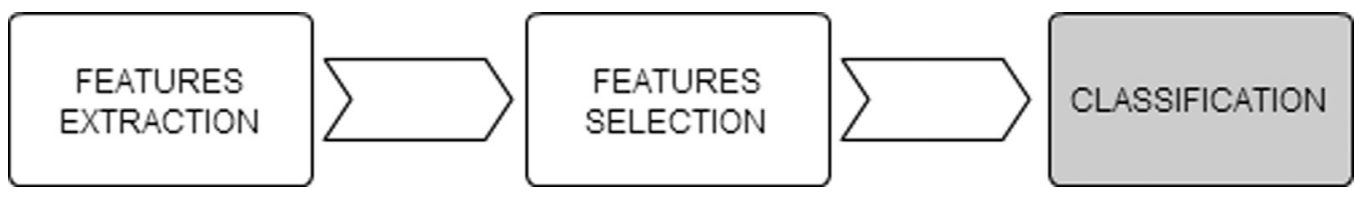

Fig. 1. Conceptual workflow of decision support systems, highlighting the focus of this systematic review.

obstructive ventilator effort is called Mixed Sleep Apnea (MSA). Moreover, hypopnea is a condition wherein the breathing is slow and shallow reducing the oxygen supply to lungs due to partial obstruction to the airway path.

The severity of apnea and hypopnea is measured by the number of episodes per hour, by means of an assessment tool, such as Apnea Index (AI), Hypopnea Index (HI), Apnea and Hypopnea Index (AHI) or Respiratory Disturbance Index (RDI). Thus, a mild case of apnea/hypopnea if observed when it happens between 5 and 15 episodes per hour, while a moderate case is verified when it occurs between 15 and 30 episodes per hour, and a severe case is a result of the occurrence of 30 or more episodes per hour.

These respiratory disturbances may lead to hypoxia and hypercapnia, which can trigger arousal from sleep by increasing ventilatory drive [2]. As a result of such sleep disruption, excessive daytime sleepiness is the most common presenting complaint [3]. Other symptoms of sleep apnea include snoring, asthma, sleep talking, sweating, chronic fatigue, falling asleep at inappropriate times of the day, morning headaches, weight gain, limited attention span, and memory loss. These symptoms can significantly decrease the quality of life and are associated with a major risk factor of health implications such as increased cardiovascular disease, sudden death, depression, irritability, hypertension, and learning difficulties [4]. Moreover, unfortunately, because of person's unawareness, SAS may go undiagnosed for years [5]. In fact, statistics show that around 100 million people worldwide are suspected to have OSA of which the vast majority undiagnosed [6]. In line with this, SAS is an important concern for public health that raises several challenges regarding it diagnosis, assessment and treatment.

The gold-standard method in SAS diagnosis is the nocturnal polysomnography (PSG). This diagnosis includes the monitoring of the breath airflow [7], snore [8], midsagittal jaw movement [9], respiratory events [10], oxygen saturation (SpO2), body position, electroencephalography (EEG), electromyography (EMG), electrooculography (EOG), and electrocardiography (ECG) which is inconvenient for a patient since it requires his connection to numerous sensors for one night, usually at the hospital under the supervision of a sleep technician. Therefore, new simplified methods and techniques for diagnosis and screening are desirable and timely. However, due to the complexity to deal with SAS symptoms, as evidenced by the multi-parameter monitoring provided by the PSG, it is advisable that new approaches could offer the ability to summarize all the collected information aiming at to extract the most relevant data, reducing the complexity of the model, before the classification process. For this reason, SAS diagnosis and screening often includes a three stages methodology as depicted in Fig. 1: feature extraction, selection of features and pattern classification. Firstly, the main purpose is to achieve a reduced set of features, extracted usually by means of algorithms from the observed data. Secondly, these features should be prioritized aiming to provide an adequate selection, which is meaningful since classification algorithms are unable to achieve high accuracy when a large number of weakly relevant and/or redundant features are managed. Thirdly, a classification method that should be wisely selected aiming to provide reasonable, reliable and consistent decisions.

In this paper, we provide a systematic review of classification methods for the decision-making on the multiple SAS scenarios, including their concepts, models, performance, plus beneficial and challenging effects.

\section{Methods}

\subsection{Research questions}

The primary research questions of this review were as follows: (RQ1) Which classification techniques have been used to support physician's decision-making on SAS? (RQ2) What is the beneficial and challenging effects stemming from the included case studies?

\subsection{Inclusion criteria}

Studies were included in this review if they met the following criteria: (1) presented a method to detect and/or to predict apnea, (2) were based on computerized systems, (3) included data about systems' evaluation, (4) presented preliminary or definitive results and (5) were written in English. These criteria were also applied to studies obtained from reference tracking. There were no age or disease restrictions; participants could be either adults or children, consisting of sleep disorders patients, whose data were collected either into a study or in a scientific database (e.g. Physionet).

\subsection{Search strategy}

To determine the state-of-the-art related with classification techniques on apnea prediction and/or detection, a search was conducted on the following databases: ACM Digital Library, and ScienceDirect. Only the studies published from the year 2005 until December 31th, 2015 and meeting the inclusion criteria were considered for this study. Two reviewers independently evaluated every study, and their suitability was determined by the agreement of both parties. A third reviewer was considered to adjudicate on differences of opinion but was not required because a consensus was reached.

\subsection{Extraction of study characteristics}

The following data were extracted from the studies and tabulated (see Table 2): year of publication, population, database, main decision, classification method, and metrics to evaluate it performance as presented on Table 1 . When a study compared several classification models the presented metrics are related to the most accurate model.

\section{Results}

The combined electronic searches identified 1537 records. Out of the 1460 titles and abstracts that were then screened to test eligibility using the PICOS screening tool (population or participant, intervention or indicator, comparator or control, outcome, and study design), 77 full-text papers were eligible for inclusion. Thus, the full text evaluation of these papers resulted in the exclusion of 37 records that did not match the defined criteria. Many of the excluded papers reported studies focus on the sleep quality and/or classification of sleep/awake events. Further hand searching 
Table 1

Outcome metrics.

\begin{tabular}{|c|c|}
\hline Metric & Definition \\
\hline Accuracy & $\frac{T P+T N}{T P+F P+T N+F N} \times 100 \%$ \\
\hline Sensitivity & $\frac{T P+T+T+F N}{T P+F N} \times 100 \%$ \\
\hline Specificity & $\frac{T N}{T N+F P} \times 100 \%$ \\
\hline Positive Predictive Value (PPV) & Ratio of true positives to total number of patients classified (correctly or incorrectly). \\
\hline Negative Predictive Value (NPV) & Ratio of true negatives to total number of patients classified (correctly or incorrectly). \\
\hline $\begin{array}{l}\text { Receiver Operating Characteristic (ROC) } \\
\text { curve }\end{array}$ & Plots of sensitivity versus 1 -Specifity \\
\hline Area under ROC curve (AUC) & $\begin{array}{l}\text { As ROC is commonly applied for comparing classifier performances because it measures how well two classes } \\
\text { are distinguished }\end{array}$ \\
\hline g-means & $\sqrt{\text { Sensitivity } \times \text { Specificity }}$ \\
\hline F1-measure & $\begin{array}{l}\text { A single measure of performance obtained as the harmonic mean of positive predictive value (precision) and } \\
\text { sensitivity (recall): } \frac{2 \times P P V \times \text { Sensitivity }}{P P V+\text { Sensitivity }}\end{array}$ \\
\hline Precision & The proportion of correctly classified positive samples to all samples classified as positive \\
\hline
\end{tabular}

TP: number of true positive cases; TN: number of true negative cases; FP: number of false positive cases; FN: number of false negative cases.
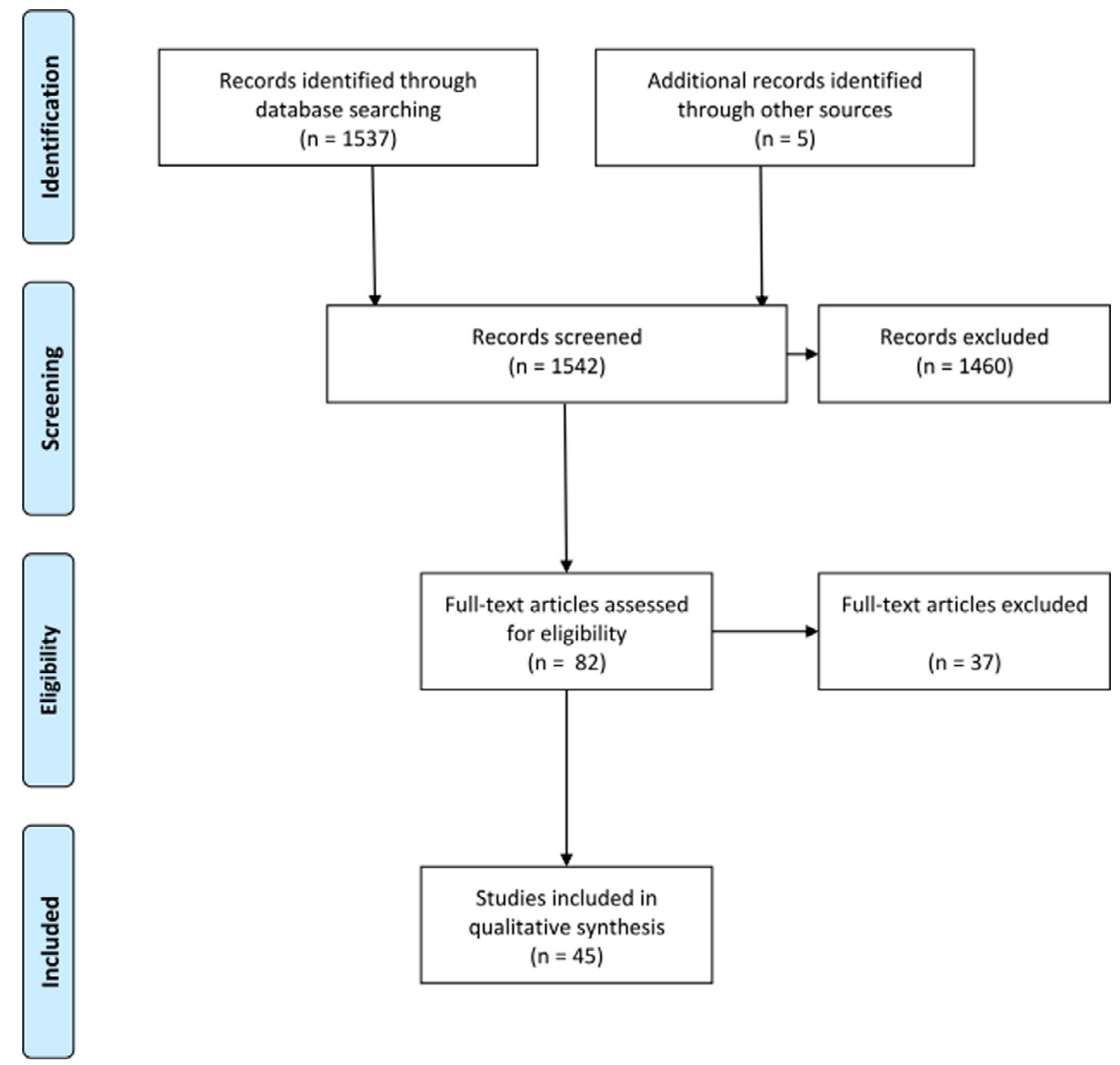

Fig. 2. Study workflow.

for reference tracking from the selected papers identify five additional studies. The selection procedure was based on the PRISMA statement which flowchart is presented in Fig. 2. In summary, our review examined 45 papers that represent 41 unique studies (some studies reported the same data and were clustered to avoid selection bias).

As shown in Table 2, all the included studies represent 1940 participants and/or subjects. Only nine of the forty-one studies (21.9\%) included in this review were published by the end of 2009. Of the remaining thirty-two studies, twenty were published by the end of 2013 (48.7\%). Finally, twelve studies (29.2\%) were published between the beginning of 2014 and the end of 2015. All of the included studies were classified as a descriptive study design; when data and characteristics about the phenomenon being examined are presented. None of the found studies neither presented a casecontrol nor a Randomized Controlled Trial (RCT). The case control means that the subjects who have a specific condition/disease (the 'cases') are compared with subjects who are similar but do not have a specific condition/disease (the 'controls'). On the contrary, in the RCT all the participants in a trial are randomly allocated either to the intervention group (under investigation) or to the control group. 
Table 2

\begin{tabular}{|c|c|c|c|c|c|c|c|c|}
\hline \multirow[t]{2}{*}{ Study } & \multirow[t]{2}{*}{ Year } & \multirow[t]{2}{*}{ Population } & \multirow[t]{2}{*}{ Decision } & \multirow{2}{*}{$\begin{array}{l}\text { Classification method }(*) \text { best when } \\
\text { multiple on the same study }\end{array}$} & \multicolumn{4}{|l|}{ Performance } \\
\hline & & & & & Ac (\%) & Se (\%) & $\mathrm{Sp}(\%)$ & Other \\
\hline Fontenla-Romero [11] & 2005 & 6 patients & OSA/MSA/CSA & Bayesian NN & $\begin{array}{l}\text { Mean (SD) } 83.78 \\
(1.90)\end{array}$ & & & \\
\hline Al-ani [12] & 2008 & $\mathrm{~N} / \mathrm{A}$ & Apnea/Non-Apnea & HMM & 70 & & & \\
\hline $\operatorname{Marcos}[13]$ & 2008 & 187 patients $(147 \mathrm{M}, 40$ F) $(57.97 \pm 12.84)$ & Apnea/Non-Apnea & ANN & 85.50 & 89.80 & 79.40 & AUC: $90.00 \%$ \\
\hline Polat [14] & 2008 & 83 patients (59 M, 24 F) [17-83 years] (mean:49) & Apnea/Non-Apnea & AIRS, ANFIS, ANN, and C4.5 $(*)$ & 95.12 & 91.67 & 96.55 & AUC: $97.00 \%$ \\
\hline Álvarez-Estévez [15] & 2009 & 12 patients & Apnea/Non-Apnea/Hypoapnea & Fuzzy Logic & & 87.00 & 89.00 & \\
\hline Khandoker $[16,17]$ & 2009 & 32 patients ( $25 \mathrm{M}, 7 \mathrm{~F})$ & Apnea/Non-Apnea & SVM & 100.00 & 100.00 & 100.00 & \\
\hline Mendez [18] & 2009 & {$[27-63$ years] $(48 \pm 10.8)$} & Apnea/Non-Apnea & ANN $(*)$, and KNN & 88.00 & 89.00 & 86.00 & \\
\hline Sezgin $[19,20]$ & 2009 & 21 patients (14 M, 7 F) [21-57 years] (mean:37) & OSA/MSA/CSA & ANN & $\begin{array}{l}\text { OA (86\%), MA (80\%), } \\
\text { CA (95\%) }\end{array}$ & & & \\
\hline Sugi [21] & 2009 & [46-58 years] & Apnea/Non-Apnea & Threshold based method & & 86.00 & & \\
\hline Gunes [22] & 2010 & 83 patients (59 M, 24 F) [17-83 years] (mean:49) & $\begin{array}{l}\text { Non-Apnea/Mild/ } \\
\text { Moderate/Serious Apnea }\end{array}$ & ANN & 84.14 & 86.37 & 74.61 & \\
\hline Ubeyli [23,24] & 2010 & 1 patient (1 M) (age:32) & Hypopnea/non-hypopnea & ANFIS $(*)$, and LS-SVM & 96.25 & 95.00 & 97.50 & \\
\hline Hsu [25] & 2011 & 15 patients (13 M, 2 F) [9-72] & Apnea/Non-Apnea & Threshold based method & 87.60 & & & \\
\hline Delibaşoğlu [26] & 2011 & $\mathrm{~N} / \mathrm{A}$ & Apnea/Non-Apnea & NARX-ANN & 96.66 & & & \\
\hline Otero [27] & 2011 & 12 patients & $\begin{array}{l}\text { Apnea / Hypopnea /Drops in } \\
\text { Sp02/ Limitations of thoracic } \\
\text { and abdominal effort }\end{array}$ & Fuzzy Set & 95.70 & & & \\
\hline Shi [28] & 2011 & $\mathrm{~N} / \mathrm{A}$ & Apnea/Non-Apnea & SVM & 91.18 & 90.02 & 91.73 & \\
\hline Tagluk [29] & 2011 & 20 patients $(36 \pm 8)$ & Apnea/Non-Apnea & ANN & 96.15 & & & \\
\hline Yildiz [30] & 2011 & 24 patients & Apnea/Non-Apnea & LS-SVM & 100.00 & 100.00 & 100.00 & \\
\hline Wang [31] & 2011 & 124 patients ( $90 \mathrm{M}, 34 \mathrm{~F}$ ) & Apnea/Non-Apnea & $\begin{array}{l}\text { Rough Set }(*) \text {, ANN, C4.5, LVQ LR, } \\
\text { and SVM }\end{array}$ & & 85.71 & 100 & g-means: $92.58 \%$ \\
\hline Al-Angari [32] & 2012 & 100 patients & Apnea/Non-Apnea & SVM & 82.40 & 69.90 & 91.40 & \\
\hline Doukas [33] & 2012 & 4 patients (2 M, 2 F) & Apnea/Non-Apnea & VAD algorithm & 98.31 & & & \\
\hline Guijarro-Berdiñas [34] & 2012 & 6 Patients & OSA/MSA/CSA & ECOC & $(90.27 \pm 0.79)$ & & & \\
\hline Martínez-Vargas [35] & 2012 & N/A & Apnea/Non-Apnea & KNN & 80.61 & 76.22 & 82.27 & \\
\hline Otero [36] & 2012 & 10 Patients & $\begin{array}{l}\text { Apnea / Hypopnea /Drops in } \\
\text { SpO2/ Limitations of thoracic } \\
\text { and abdominal effort }\end{array}$ & Fuzzy Set & 90.00 & & & \\
\hline Alencar [37] & 2013 & 17 patients $(11 \mathrm{M}, 6 \mathrm{~F})(50 \pm 10)$ & Apnea/Non-Apnea & Hurst's R/S based algorithm & 94.11 & & & \\
\hline Almazaydeh [38] & 2013 & 100 healthy people & Apnea/Non-Apnea & VAD algorithm & 97.00 & & & \\
\hline Jafari [39] & 2013 & $\mathrm{~N} / \mathrm{A}$ & Apnea/Non-Apnea & SVM & 94.80 & 94.16 & 95.42 & \\
\hline LaFleur $[40]$ & 2013 & $\mathrm{~N} / \mathrm{A}$ & Apnea/Non-Apnea & LDA & & 99.74 & & PPV: 99.80 \\
\hline Maali [41] & 2013 & 5 patients & Apnea/Non-Apnea & $\operatorname{BPNN}\left({ }^{*}\right)$, RBFNN, and SRN & & & & AUC: $87 \%$ \\
\hline Zhang [42] & 2013 & 40 Patients & Apnea/Non-Apnea & SVM & 94.37 & 95.71 & 89.47 & \\
\hline Al-Mardini [43] & 2014 & 15 patients (14 M, $1 \mathrm{~F}$ ) & Apnea/Non-Apnea & Score (Apnea/Hypopnea Index, AHI) & 87.50 & 100 & 85.50 & $\begin{array}{l}\text { PPV: } 99.90 \% \\
\text { NPV: } 100 \%\end{array}$ \\
\hline Benavides [44] & 2014 & 82 patients ( $82 \mathrm{M}, 0 \mathrm{~F}$ ) & Apnea/Non-Apnea & LDA, and Multi-linear Regression & & 85.00 & 75.00 & $\begin{array}{l}\text { PPV: } 77.30 \% \text { NPV: } \\
\text { F3.30\% } \\
\text { F1-measurement: }\end{array}$ \\
\hline Erazo [45] & 2014 & $\mathrm{~N} / \mathrm{A}$ & Apnea/Non-Apnea & ANN $(*)$, and SVM & 55.94 & 84.36 & 19.00 & \\
\hline Sánchez-Morillo [46] & 2014 & 115 patients $(83 \mathrm{M}, 32 \mathrm{~F})(58.40 \pm 13.40)$ & $\begin{array}{l}\text { Non-Apnea/Mild / Moderate } \\
\text { /Serious Apnea }\end{array}$ & $\begin{array}{l}\text { Binary Hierarchical Classification } \\
\text { structure (BHC) using: Decision Tree, }\end{array}$ & 79.30 & 76.70 & 82.60 & $\begin{array}{l}\text { Precision: } 85.20 \% \\
\text { F1-measurement: } \\
90 \% \text { AUC. } 90 \%\end{array}$ \\
\hline Solé-Casals [47] & 2014 & 376 patients (253 M, 123 F) [18-82] & Apnea/Non-Apnea & $\begin{array}{l}\text { AdaBoost, ANN, KNN, SVM, and } \\
\text { Bayesian classification }\left(^{*}\right)\end{array}$ & 82.04 & 81.74 & 82.40 & \\
\hline Travieso [48] & 2014 & $\mathrm{~N} / \mathrm{A}$ & Apnea/Non-Apnea & $\mathrm{HMM}+\mathrm{SVM}$ & 99.23 & & & \\
\hline Abdelnasser [49] & 2015 & 3 patients & Apnea/Non-Apnea & Threshold based method & 96.00 & & & \\
\hline Cohen [50] & 2015 & 394 patients (193 M, $201 \mathrm{~F}$ ) & Apnea/Non-Apnea & LDA & 66.70 & 58.10 & 67.00 & \\
\hline Hassan [51] & $\begin{array}{r}2015 \text { (on } \\
\text { press) }\end{array}$ & {$[27-63$ years] $(45 \pm 10)$} & Apnea/Non-Apnea & Bagging & 85.97 & 84.14 & 86.83 & \\
\hline Lee [52] & 2015 & $\mathrm{~N} / \mathrm{A}$ & OSA/CSA/ Hypopnea & Adaboost & 85.00 & 90.00 & 80.00 & AUC: $84.00 \%$ \\
\hline Nandakumar $[53,54]$ & 2015 & 37 patients (20 M, 17 F) [23-93] & OSA/MSA/CSA/Hypoapnea & Peak detection algorithm & $\begin{array}{l}\text { Mean (SD) } 97.83 \\
(2.22)\end{array}$ & & & \\
\hline Samy [55] & 2015 & 16 patients & Apnea/Non-Apnea & KNN, and SVM $(*)$ & 79.80 & 66.7 & 83 & \\
\hline
\end{tabular}


On the one hand, as presented in Table 3, the 41 unique studies revealed a combination of classifiers that were clustered as follows: threshold-based methods (14.75\%), such as: Voice Activity Detection (VAD), peak detection methods, and combined thresholds, and machine learning (ML) methods (85.25\%) segmented as follows: neural networks (44.26\%), regression (4.91\%), instancebased $(11.47 \%)$, reinforcement learning (4.91\%), ensemble learning (6.55\%), decision trees (3.27\%), dimensionality reduction $(8.19 \%)$, and Bayesian model (1.63\%).

On the other hand, as presented in Table 3, the decision making is based on the following scenarios: binary decision, such as: apnea or non-apnea and hypoapnea or non-hypoapnea, presented fifty-one times (83.59\%), three-option decision, such as: apnea, or non-apnea or hypopnea, presented four times (6.55\%), or four and more option decision presented six times (9.83\%). When the decision making is combined with the classifier model, was observed that the neural networks to detect apnea or non-apnea was the most popular scenario considered in twenty two studies (53.65\%).

In the next sections, we briefly describe the principles of threshold for classification, and several ML techniques are presented including a conceptual mind-map as depicted in Fig. 3, closing with the observed beneficial and challenging effects as a result of a fusion between the clinical and the computer science perspective.

\subsection{Threshold-based classification}

The threshold-based classification is operate using different limits, typically support and confidence thresholds, and thus their operation is very much dependent on the selection of appropriate values [56]. The observed methods and its description are detailed in Table 4.

\subsection{Machine learning classification}

The ML has revolutionized the possibility to deal with large and complex electronic data sets [57]. Nevertheless, rapid technological developments continue to pose several challenges for data scientists to ensure computational models with the ability not only to cope with big data, and multidimensional data structures, but also to provide accurate and reliable performances. In the next sub-sections, different machine learning techniques are briefly described.

\subsubsection{Neural networks}

Artificial neural networks (ANNs) aim to perform tasks analogous to biological brains based on the connections among many simple processing elements, so called neurons. Their main feature is the ability to change their behavior based on the external information that flows through an ANN during the learning (training) phase. Neurons are organized into layers, where outputs from one layer are used as inputs into the following layer. However, the amount of the measurement data of the network output is proportional to its size. This means that the complexity of the tasks to be accomplished leads to huge data, and this places a great deal of burden on transmission networks of limited capacity. Other neural networks techniques were observed in this review, such as, the Bayesian neural network (BNN) [58], the Adaptive neuro-fuzzy inference system (ANFIS) [59], the Simple recurrent network (SRN) [60], the Radial basis function neural network (RBFNN) [61], the Support vector machine (SVM) [62], the Least squares SVM [63], and the Probabilistic neural networks (PNN). The BNN aims to cast the training phase as a problem of inference, which is solved using Bayes' theorem. On the contrary, the ANFIS is functionally equivalent to a fuzzy inference system which uses a hybrid learning algorithm. In fact, this model is based on a network, to manage

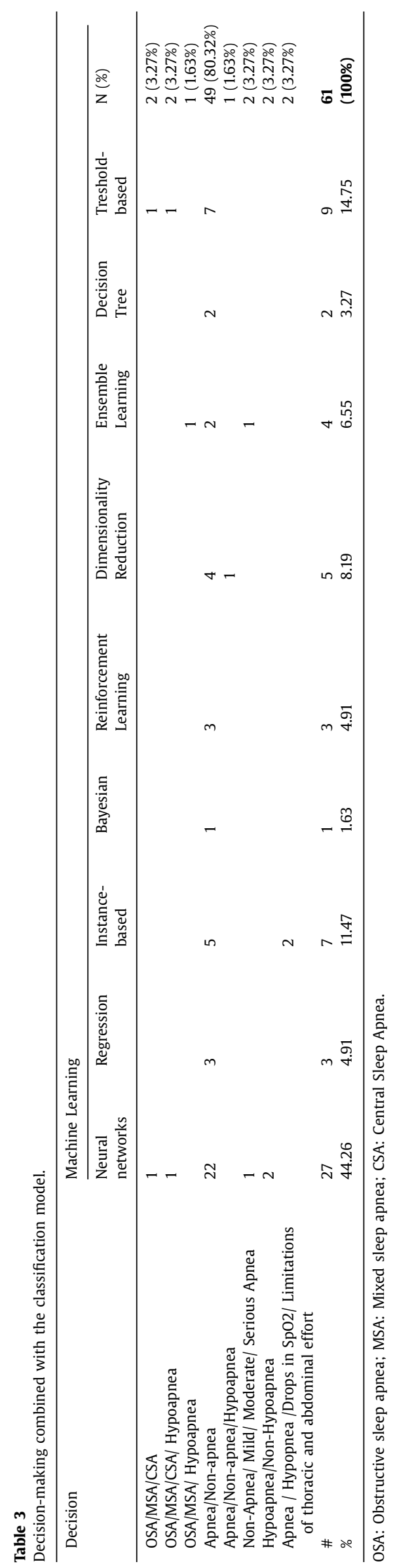




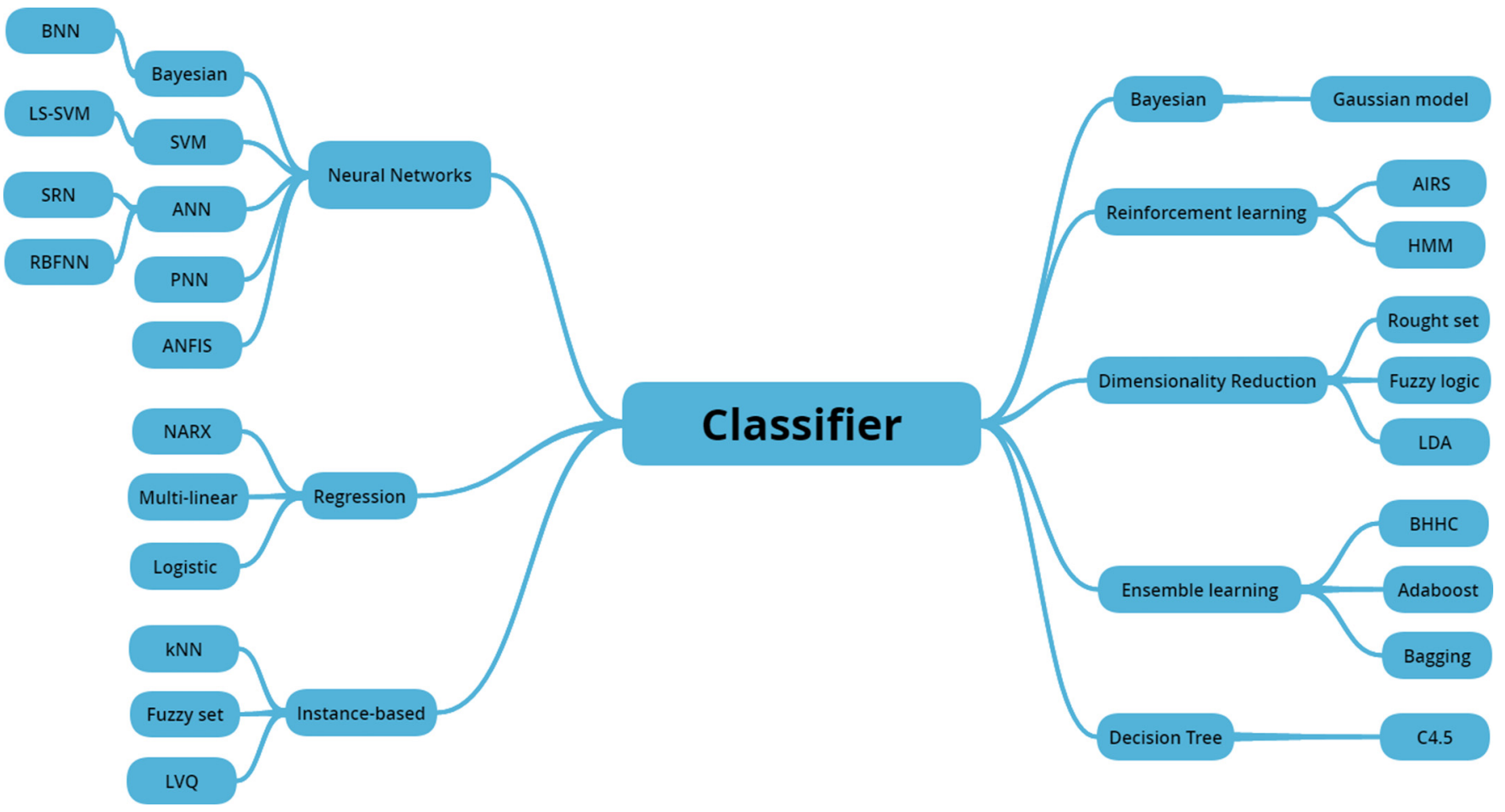

Fig. 3. Mind map of the observed machine learning techniques.

Table 4

Threshold-based methods.

\begin{tabular}{|c|c|}
\hline Study & Description \\
\hline Abdelnasser [49] & Threshold value for detecting the significantly increase and/or decrease in the breathing signal. \\
\hline Hsu [25] & $\begin{array}{l}\text { Combined threshold values for detecting the significantly increase and/or decrease in the delta wave ratio on the EEG } \\
\text { signal, and therefore the duration of apnea or hypopnea events. }\end{array}$ \\
\hline Sugi [21] & Combined threshold values for detecting EEG arousals in the PSG record for detect sleep apnea events. \\
\hline Doukas [33], Almazaydeh [38] & VAD for modelling of snore signal. \\
\hline Al-Mardini $[43]$ & $\begin{array}{l}\text { Combined values such as body movement, audio record on respiratory efforts, and oxygen level to obtain the average } \\
\text { number of apnea events per hour. The severity of the apnea is determined as follows: (1) mild when } 5 \leq \text { average }< \\
15 \text {, (2) moderate when } 15 \leq \text { average }<30 \text {, (3) and severe when average } \geq 30 \text {. }\end{array}$ \\
\hline Alencar [37] & $\begin{array}{l}\text { Model based on the Hurst's R/S principles, namely considering the time intervals between snore events, and the } \\
\text { average number of snore events per unit time. }\end{array}$ \\
\hline Nandakumar $[53,54]$ & $\begin{array}{l}\text { Peak detection algorithm for identify the transitions points at which the EEG signal changes from an increasing to a } \\
\text { decreasing trend. }\end{array}$ \\
\hline
\end{tabular}

fuzzy data, combined with an inference model. The SRN is a typical ANN with an additional set of units, called context units aiming to provide recurrence on the network. The RBFNN is commonly used for modeling nonlinear problems and presents one hidden layer of nodes that perform a fixed nonlinear transformation with no adjustable parameters and it maps the input space onto a new space, and a network output obtained from the linear combination of weighted with connecting weights [64]. The SVM attempts to determine the tradeoff between minimizing the training set error and maximizing the margin in order to achieve the best generalization characteristics that may lead to trustworthy decisions. In addition, LS-SVM, aims to solve linear equations by using equality constraints and a least squares loss function which greatly reduces the computational complexity [65]. Finally, the PNN approximates the class-conditional probability distributions by finite mixtures of product components under the assumption that probability distributions can be estimated.

\subsubsection{Regression}

Regression is the process of learning relationships between inputs and continuous outputs from example data, which enables predictions for novel inputs. Several techniques were observed such as, the nonlinear autoregressive network with exogenous inputs (NARX) [66], the Logistic regression (LR) [67], and the Multilinear regression [68] (MLR). The NARX, is used for time series prediction and modeling, in particular, when they are noisy, and represent a nonlinear system not easily approached through analytical means. The LR is used to analyze the relationship between predictors, and an outcome that is dichotomous responses such as the presence or absence of an apnea event. In addition, the MLR is commonly used to model the impact of numerous independent factors on the dependent outcome being examined.

\subsubsection{Instance-based}

Such methods typically build up an instance of training data and compare new data to the instance using a similarity measure in order to find the best match and make a prediction. The kNearest neighbor (kNN) [69], the Fuzzy set [70], and the Learning vector quantization (LVQ) [71] were the observed instance-based techniques. The kNN predicts objects "values" or class memberships based on the $k$ closest training samples in the feature space, and selected according to the similarity metrics such as: Euclidean distance, or Manhattan distance. On the contrary, the LVQ it heavily relies on the possibility to pick instances as members of the 
data space and to adapt these representatives smoothly by means of vectorial updates triggered by the data. The Fuzzy set aims to represent uncertainty and vagueness and to formalize tools for dealing with the fuzzy logic.

\subsubsection{Bayesian algorithms}

The classical Bayesian classifier consists of the following steps: identification of the number of classes, fitting a distribution (e.g. Gaussian) to the histogram of the selected outer beams, and assigning classes to the rest of the beam angles according to the distribution. The observed model is related with a multivariate normal model for the distribution of each class [47].

\subsubsection{Reinforcement learning}

Reinforcement learning models formalize the process through which stimulus-reward predictions are acquired and used to guide choice behavior. Two techniques were observed such as the Hidden Markov Model (HMM) [72] and the Artificial immune recognition system (AIRS) [73]. On the one hand, the HMM can express complex Markov processes where a finite set of discrete unobserved (hidden) states emit the observations according to some probability distribution. On the other hand, the AIRS is a resource limited algorithm, in which the used immune mechanisms are resource competition, clonal selection, affinity maturation and memory cell formation [14].

\subsubsection{Dimensionality reduction}

Dimensionality reduction models aims to summarize or describe data using less information. Three techniques were observed such as the Linear discriminant analysis (LDA) [74], the Rough set [75] and the Fuzzy logic [70]. The LDA finds the direction vector such that projected data have the largest possible between-class separation while the within-class variance is kept as small as possible. On the contrary, the Rough set comprises a combination of two sets: the lower and the upper approximation. The lower approximation is made up of elements that definitely belong to the set, whereas the upper approximation is composed of elements that possibly belong to the set. The difference between them defines the boundary region of the rough set [76]. The Fuzzy logic represents a possibility logic model that uses reasoning to explain whether an event is about to happen.

\subsubsection{Ensemble learning}

Ensemble methods direct to train multiple independent models, and to combine its predictions aiming to obtain the overall prediction. Several techniques were observed such as, the Adaboost [77], the Bagging [78], the Binary hierarchical classification structure (BHC) [79], and the error correcting output code (ECOC). The Adaboost, focuses on learning the misclassified examples and trains classifiers in an iterative way, in order to obtain a final decision based on the weighted majority voting rule for each classifier. The Bagging directs to address potential instabilities stem from sources such as either data or parameter uncertainty in order to obtain a suitable predictor. The BHC aims to combine different classification models in a top-down or bottom-up hierarchy. Finally, the ECOC aims to build a set of classifiers that involves the manipulation of the desired outputs representing the classification of each sample in the training set.

\subsubsection{Decision tree}

These models train on data for classification and regression problems based on decisions fork in tree structures. The C4.5 algorithm uses a measure of information gain ratio for selecting an input variable in each node. This variable selection process is based on the precise probabilities calculated from the training set.

\subsection{Beneficial and challenging effects}

The efficacy of the selected features and its effects on the classifiers' accuracy is highlighted [18,25,26,28,30,32,34,35,44,51,52,55], as well as the opportunity for the design of outperformed models based on a multi-signal combination $[16,17,45,50]$. In addition, the application of wavelets in extracting features was found to allow better quantification of the frequency components associated with the SAS $[16,17,19]$. On the contrary, the combination of classifiers is also appreciated $[46,48]$. The validation of the model using a large sample of data and/or additional databases is an observed need $[13,34,37,48,51]$. The challenge for the development of reliable, minimally invasive, and ubiquitous systems for use in home settings as a solid alternative to the PSG, the standard clinical test for SAS in medical settings, is observed $[37,39,42,50,53,54]$.

On the one hand, threshold-based methods revealed its suitability to detect discrepancies, and abnormalities in the signal processing, such as ECG, EEG, EMG, among others. However an efficient calibration is required in order to enable these models for different severities of apnea [25,44].

On the other hand, related with ML technologies, the overfitting raised by complex models for the classification of apnea conditions is identified [47]. On the contrary, when inflexible models lead to underfitting is also an observable limitation [13]. Ideally, a model should provide an auto-adaptive and no external-human action dependency [11].

Moreover, authors in $[21,23,24,29,33,46]$ suggested that the implementation of computerized systems may reduce the cost and time needed for an accurate diagnosis. Only two studies; [41,55], exhibit a predictive model for the apnea condition while the remaining are focused to detect apnea events, therefore, for diagnosis and/or screening purposes. Finally, the clinical acceptance of all the included studies is limited because were simply not designed as an RCT.

\section{Discussion}

In this study, we presented an overview of the classification techniques on the prediction and/or detection of SAS by conducting a systematic review study on articles written during 20052015. A multi-step process was performed to select the studies, i.e. they were searched for in databases by using terms to identify related studies; some were excluded based on the criteria of exclusion, while relevant studies were included based on analysis of their titles and abstracts. Finally, the case studies were obtained based on full text reading. From a high-level overview, the observed classification techniques fell into two thematic categories of models, such as threshold-based, and ML. On the one hand, as presented in Table 4, when the identification of the categories relies on several parameters thresholds, which require an appropriate selection of values, is called by threshold-based classification. On the other hand, in this study, we presented a mind map (see Fig. 3) related with the different ML techniques observed on computerized systems on SAS. The ML were clustered as follows: neural networks, regression, instance-based, Bayesian algorithms, reinforcement learning, dimensionality reduction, ensemble learning, and decision trees.

The most common scenario observed is based on neural networks. There are several reasons for which this may be considered the method of choice, including the ability to extract complex relations in the problem domain [80-82]. However, this methodology is limited in terms of knowledge visualization. Additionally, determining the adequate size of the hidden layer is vulnerable to poor approximations (caused by lack of nodes) and overfitting (from excessive nodes) [76]. 
As ML techniques are highly dependent on the underlying data, research which uses highly specific datasets which are confined to small samples of cases from distinct populations suffers from limitations of generalizability of results. Thus, further research is needed to validate the generality of SAS models on a larger dataset or within different populations. On the contrary, hybrid approaches (presented only in two studies $[46,48]$ ) try to combine two or more algorithms aiming to enhance the performance, remains understudied and thus further research is needed to validate the suitability of these models on SAS.

Moreover, since medical systems should have especial characteristics such as high accuracy and reliability then clinical validation is highly recommended, for example using RCTs study design. In fact, the effect of various classification models on algorithm's performance, remains poorly explored.

Finally, due to the fact that the PSG requires an exhaustive test in a hospital setting, high cost and discomfort to the patient, is observable an effort to implement a non-invasive, accurate and home-bound techniques based on a reduced and simplified set of biometric signals.

\section{Conclusions and future directions}

This systematic literature review has synthesized and summarized the existing classification techniques on the prediction and/or detection of SAS. Forty-five studies were examined and the main findings are summarized as follows:

- (RQ1) the classification techniques have been used physician's decision-making to support the SAS include threshold-based and machine learning models, which were clustered into neural networks, regression, instance-based, Bayesian algorithms, reinforcement learning, dimensionality reduction, ensemble learning, and decision trees. All these models were combined in a mind map (see Fig. 3).

- (RQ2) The need for validation of the models either from the clinical perspective using a randomized controlled trial, or from the computer science perspective using a large and/or multiple sample of data. In addition, the accuracy of the classification models is related with the effective features selection.

The growth in ML approaches in SAS is encouraging and studies show promising results and useful applications. However, from a ML perspective, there is a need for predictive models and for further comparative studies, which may evaluate the accuracy and applicability of a range of ML approaches in different contexts. Furthermore, recent studies using either nonlinear and nonstationary time series analysis, or a reduced volume of data revealed its feasibility and suitability to deal with apnea symptoms [83-88]. Therefore, complementary studies should be addressed not only to evaluate the effect between the extraction and selection of features, and the accuracy of the classification model, but also to design innovative models using time series principles whose roadmap may include further research on either stochastic nonlinear time-delay systems $[89,90]$ or combined fault-tolerant with predictive control [91,92].

Moreover, the design of reliable, minimally invasive, and ubiquitous systems for use in home settings as a solid alternative to the PSG, the standard clinical test for SAS in medical settings, is both promising and challenging.

Finally, some limitations of this review should be mentioned. First, the studies included in this review presented diverse participants/subjects and different clinical contexts of the apnea condition, meaning that assumptions about the generalizability of the findings to the target population should be made with some caution. For this reason, comparisons between studies are also unsuitable. Second, every included study represents a non-randomized controlled trial, which increases the heterogeneity and the risk of the selection bias of this review. Third, some studies did not report clearly on the data that are used for SAS (e.g. the performance metrics were not presented). Four, only English-language publications were included.

\section{Acknowledgments}

This work was supported by FCT project UID/EEA/50008/2013 (Este trabalho foi suportado pelo projecto FCT UID/EEA/50008/ 2013). The authors would also like to acknowledge the contribution of the COST Action IC1303 - AAPELE - Architectures, Algorithms and Protocols for Enhanced Living Environments.

\section{References}

[1] Listed $\mathrm{N}$ authors, Sleep-related breathing disorders in adults: recommendations for syndrome definition and measurement techniques in clinical research. The report of an american academy of sleep medicine task force, Sleep. 22 (August(5)) (1999) 667-689.

[2] K. Gleeson, C.W. Zwillich, D.P. White, The influence of increasing ventilatory effort on arousal from sleep, Am Rev Respir Dis 142 (August(2)) (1990) 295-300.

[3] G. Slater, J. Steier, Excessive daytime sleepiness in sleep disorders, J Thoracic Dis 4 (December(6)) (2012) 608-616.

[4] N. Ben-Israel, A. Tarasiuk, Y. Zigel, Nocturnal sound analysis for the diagnosis of obstructive sleep apnea, in: Engineering in medicine and biology society (EMBC), 2010 annual international conference of the IEEE, 2010, pp. 6146-6149.

[5] M.H. Kryger, L. Roos, K. Delaive, R. Walld, J. Horrocks, Utilization of health care services in patients with severe obstructive sleep apnea, Sleep 19 (November(9)) (1996) S111-S116.

[6] J. Bousquet, N.G. Khaltaev, A.A. Cruz, W.H. Organization, Global surveillance, prevention and control of chronic respiratory diseases: a comprehensive approach [Internet], World Health Organization, 2007 (Nonserial Publication). Available from:https://books.google.pt/books?id=gdj5iU5FrXEC.

[7] P. Lloberes, S. Sánchez-Vidaurre, À. Ferré, M.J. Cruz, J. Lorente, G. Sampol, et al., in: Effect of continuous positive airway pressure and upper airway surgery on exhaled breath condensate and serum biomarkers in patients with sleep apnea, 50, Archivos de Bronconeumología, 2014, pp. 422-428.

[8] A. Azarbarzin, Z. Moussavi, Snoring sounds variability as a signature of obstructive sleep apnea, Med Eng Phys 35 (4) (2013) 479-485.

[9] F. Senny, J. Destine, R. Poirrier, Midsagittal jaw movement analysis for the scoring of sleep apneas and hypopneas, IEEE Trans Biomed Eng 55 (January(1)) (2008) 87-95.

[10] R.G. Norman, M.M. Ahmed, J.A. Walsleben, D.M. Rapoport, Detection of respiratory events during NPSG: nasal cannula/pressure sensor versus thermistor, Sleep 20 (December(12)) (1997) 1175-1184.

[11] O. Fontenla-Romero, B. Guijarro-Berdiñas, A. Alonso-Betanzos, V. Moret-Bonillo, A new method for sleep apnea classification using wavelets and feedforward neural networks, Artif Intell Med 34 (1) (2005) 65-76.

[12] T. Al-ani, C.K. Karmakar, A.H. Khandoker, M. Palaniswami, Automatic recognition of obstructive sleep apnoea syndrome using power spectral analysis of electrocardiogram and hidden Markov Models, in: Intelligent sensors, sensor networks and information processing, 2008 ISSNIP 2008 international conference on, 2008, pp. 285-290.

[13] J.V. Marcos, R. Hornero, D. Álvarez, Fdel Campo, C. Zamarrón, M. López, Utility of multilayer perceptron neural network classifiers in the diagnosis of the obstructive sleep apnoea syndrome from nocturnal oximetry, Comput Methods Program Biomed 92 (1) (2008) 79-89.

[14] K. Polat, Ş. Yosunkaya, S. Güneș, Comparison of different classifier algorithms on the automated detection of obstructive sleep apnea syndrome, J Med Syst 32 (June(3)) (2008) 243-250.

[15] D. Álvarez-Estévez, V. Moret-Bonillo, Fuzzy reasoning used to detect apneic events in the sleep apnea-hypopnea syndrome, Expert Syst Appl 36 (4) (2009) 7778-7785.

[16] A.H. Khandoker, C.K. Karmakar, M. Palaniswami, Automated recognition of patients with obstructive sleep apnoea using wavelet-based features of electrocardiogram recordings, Comput Biol Med 39 (1) (2009) 88-96.

[17] A.H. Khandoker, M. Palaniswami, C.K. Karmakar, Support vector machines for automated recognition of obstructive sleep apnea syndrome from ECG recordings, Inf Technol Biomed IEEE Trans 13 (January(1)) (2009) 37-48.

[18] M.O. Mendez, A.M. Bianchi, M. Matteucci, S. Cerutti, T. Penzel, Sleep apnea screening by autoregressive models from a single ECG lead, Biomed Technol IEEE Trans 56 (December(12)) (2009) 2838-2850.

[19] N. Sezgin, M.E. Tagluk, Energy based feature extraction for classification of sleep apnea syndrome, Comput Biol Med 39 (11) (2009) 1043-1050.

[20] M.E. Tagluk, M. Akin, N. Sezgin, Classıfication of sleep apnea by using wavelet transform and artificial neural networks, Expert Syst Appl 37 (2) (2010) $1600-1607$.

[21] T. Sugi, F. Kawana, M. Nakamura, Automatic $\{\mathrm{EEG}\}$ arousal detection for sleep apnea syndrome, Biomed Signal Process Control 4 (4) (2009) 329-337. 
[22] S. Güneș, K. Polat, Ş. Yosunkaya, Multi-class f-score feature selection approach to classification of obstructive sleep apnea syndrome, Expert Syst Appl 37 (2) (2010) 998-1004.

[23] E.D. Übeyli, D. Cvetkovic, G. Holland, I. Cosic, Analysis of sleep $\{$ EEG $\}$ activity during hypopnoea episodes by least squares support vector machine employing \{AR\} coefficients, Expert Syst Appl 37 (6) (2010) 4463-4467.

[24] E.D. Übeyli, D. Cvetkovic, G. Holland, I. Cosic, Adaptive neuro-fuzzy inference system employing wavelet coefficients for detection of alterations in sleep \{EEG\} activity during hypopnoea episodes, Digital Signal Process 20 (3) (2010) 678-691.

[25] C.-C. Hsu, P.-T. Shih, A novel sleep apnea detection system in electroencephalogram using frequency variation, Expert Syst Appl 38 (5) (2011) 60146024.

[26] I. Delibaş oğ lu, C. Avci, A. Akbaș, ECG based sleep apnea detection using wavelet analysis of instantaneous heart rates, in: Proceedings of the 4th international symposium on applied sciences in biomedical and communication technologies [Internet], ACM, New York, NY, USA, 2011, p. 40. http://doi.acm. org/10.1145/2093698.2093738.

[27] A. Otero, P. Félix, M.R. Álvarez, Algorithms for the analysis of polysomnographic recordings with customizable criteria, Expert Syst Appl 38 (8) (2011) 10133-10146.

[28] C. Shi, M. Nourani, G. Gupta, L. Tamil, A virtual sleep laboratory, in: Proceedings of the first ACM workshop on mobile systems, applications, and services for healthcare [Internet], ACM, New York, NY, USA, 2011, p. 10. http: //doi.acm.org/10.1145/2064942.2064956.

[29] M.E. Tagluk, N. Sezgin, A new approach for estimation of obstructive sleep apnea syndrome, Expert Syst Appl 38 (5) (2011) 5346-5351.

[30] A. Yildiz, M. Akı n, M. Poyraz, An expert system for automated recognition of patients with obstructive sleep apnea using electrocardiogram recordings, Expert Syst Appl 38 (10) (2011) 12880-12890.

[31] P.-C. Wang, C.-T. Su, K.-H. Chen, N.-H. Chen, The application of rough set and Mahalanobis distance to enhance the quality of $\{\mathrm{OSA}\}$ diagnosis, Expert Syst Appl 38 (6) (2011) 7828-7836.

[32] H.M. Al-Angari, A.V. Sahakian, Automated recognition of obstructive sleep apnea syndrome using support vector machine classifier, Inf Technol Biomed IEEE Trans 16 (May(3)) (2012) 463-468.

[33] C. Doukas, T. Petsatodis, C. Boukis, I. Maglogiannis, Automated sleep breath disorders detection utilizing patient sound analysis, Biomed Signal Process Control 7 (3) (2012) 256-264

[34] B. Guijarro-Berdiñas, E. Hernández-Pereira, D. Peteiro-Barral, A mixture of experts for classifying sleep apneas, Expert Syst Appl 39 (8) (2012) $7084-$ 7092.

[35] J.D. Martínez-Vargas, L.M. Sepulveda-Cano, C. Travieso-Gonzalez, G. Castellanos-Dominguez, Detection of obstructive sleep apnoea using dynamic filter-banked features, Expert Syst Appl 39 (10) (2012) 9118-9128.

[36] A. Otero, P. Félix, S. Barro, C. Zamarrón, A structural knowledge-based proposal for the identification and characterization of apnoea episodes, Appl Soft Comput 12 (1) (2012) 516-526.

[37] A.M. Alencar, D.G.V.da Silva, C.B. Oliveira, A.P. Vieira, H.T. Moriya, G. LorenziFilho, Dynamics of snoring sounds and its connection with obstructive sleep apnea, Physica A Statis Mech Appl 392 (1) (2013) 271-277.

[38] L. Almazaydeh, K. Elleithy, M. Faezipour, A. Abushakra, Apnea detection based on respiratory signal classification, Procedia Comput Sci 21 (2013) 310-316.

[39] A. Jafari, Sleep apnoea detection from $\{$ ECG $\}$ using features extracted from reconstructed phase space and frequency domain, Biomed Signal Process Control 8 (6) (2013) 551-558.

[40] S. LaFleur, I. Mahgoub, A personal body area network as a pre-screening surrogate to the polysomnography, in: Proceedings of the 8th international conference on body area networks [Internet]. ICST, Brussels, Belgium, Belgium: ICST (Institute for Computer Sciences, Social-Informatics and Telecommunications Engineering), 2013, pp. 233-236. http://dx.doi.org/10.4108/icst.bodynets.2013. 253687.

[41] Y. Maali, A. Al-Jumaily, Multi neural networks investigation based sleep apnea prediction, Procedia Computer Science 24 (2013) 97-102.

[42] J. Zhang, Q. Zhang, Y. Wang, C. Qiu, A real-time auto-adjustable smart pillow system for sleep apnea detection and treatment, in: Proceedings of the 12th international conference on information processing in sensor networks [Internet], ACM, New York, NY, USA, 2013, pp. 179-190. http://doi.acm.org/10.1145/ 2461381.2461405.

[43] M. Al-Mardini, F. Aloul, A. Sagahyroon, L. Al-Husseini, Classifying obstructive sleep apnea using smartphones, J Biomed Inf 52 (2014) 251-259.

[44] A.M. Benavides, R.F. Pozo, D.T. Toledano, J.L.B. Murillo, E.L. Gonzalo, L.H. Gómez, Analysis of voice features related to obstructive sleep apnoea and their application in diagnosis support, Comput Speech Language 28 (2) (2014) 434-452.

[45] L. Erazo, S.A. Ríos, A benchmark on automatic obstructive sleep apnea screening algorithms in children, Procedia Comput Sci 35 (2014) 739-746.

[46] D. Sánchez-Morillo, M.A. López-Gordo, A. León, Novel multiclass classification for home-based diagnosis of sleep apnea hypopnea syndrome, Expert Syst Appl 41 (4, Part 2) (2014) 1654-1662.

[47] J. Solé-Casals, C. Munteanu, O.C. Martín, F. Barbé, C. Queipo, J. Amilibia, et al., Detection of severe obstructive sleep apnea through voice analysis, Appl Soft Comput 23 (2014) 346-354.

[48] C.M. Travieso, J.B. Alonso, M.del Pozo, J.R. Ticay, G. Castellanos-Dominguez, Building a cepstrum-HMM kernel for apnea identification, Neurocomputing 132 (2014) 159-165.
[49] H. Abdelnasser, K.A. Harras, M. Youssef, UbiBreathe: a ubiquitous non-invasive WiFi-based breathing estimator, in: Proceedings of the 16th ACM international symposium on mobile ad hoc networking and computing [Internet], New York, NY, USA, ACM, 2015, pp. 277-286. http://doi.acm.org/10.1145/2746285. 2755969.

[50] G. Cohen, P.de Chazal, Automated detection of sleep apnea in infants: a multi-modal approach, Comput Biol Med 63 (2015) 118-123.

[51] A.R. Hassan, M.A. Haque, Computer-aided obstructive sleep apnea screening from single-lead electrocardiogram using statistical and spectral features and bootstrap aggregating, Biocybern Biomed Eng (2015).

[52] J.-H. Lee, H.-J. Park, Y.-N. Kim, Monitoring obstructive sleep apnea with electrocardiography and 3-axis acceleration sensor, in: Proceedings of the 8th ACM international conference on pervasive technologies related to assistive environments [Internet], New York, NY, USA, ACM, 2015, p. 74. http://doi.acm.org/10. $1145 / 2769493.2769522$.

[53] R. Nandakumar, S. Gollakota, N. Watson, Contactless sleep apnea detection on smartphones, in: Proceedings of the 13th annual international conference on mobile systems, applications, and services [Internet], New York, NY, USA, ACM, 2015, pp. 45-57. http://doi.acm.org/10.1145/2742647.2742674.

[54] R. Nandakumar, S. Gollakota, N. Watson, Contactless sleep apnea detection on smartphones, GetMobile: Mobile Comp and Comm 19 (3) (2015 Dec) 22-24.

[55] L. Samy, P.M. Macey, N. Alshurafa, M. Sarrafzadeh, An automated framework for predicting obstructive sleep apnea using a brief, daytime, non-intrusive test procedure, in: Proceedings of the 8th ACM international conference on pervasive technologies related to assistive environments [Internet], New York, NY, USA, ACM, 2015, p. 70. http://doi.acm.org/10.1145/2769493.2769541.

[56] F. Coenen, P. Leng, The effect of threshold values on association rule based classification accuracy, Data Knowl Eng 60 (2) (2007) 345-360.

[57] B. Hammer, B. Mokbel, F.-M. Schleif, X. Zhu, Prototype-based classification of dissimilarity data, in: J Gama, E Bradley, J Hollmén (Eds.), Advances in intelligent data analysis X: 10th international symposium, IDA 2011, Porto, Portugal, October 29-31, 2011 proceedings [Internet], Springer Berlin Heidelberg, Berlin, Heidelberg, 2011, pp. 185-197. http://dx.doi.org/10.1007/978-3-642-24800-9_ 19.

[58] D.J.C.A. MacKay, Practical Bayesian framework for backpropagation networks, Neural Comput 4 (May(3)) (1992) 448-472.

[59] J.S.R. Jang, ANFIS: adaptive-network-based fuzzy inference system, IEEE Trans Syst Man Cybern 23 (May(3)) (1993) 665-685.

[60] J.L. Elman, Distributed representations, simple recurrent networks, and grammatical structure, Mach Learn 7 (September(2-3)) (1991) 195-225.

[61] S. Haykin, Neural networks: a comprehensive foundation, 2nd ed., Prentice Hall PTR, Upper Saddle River, NJ, USA, 1998.

[62] V.N. Vapnik, in: M Jordan, SLe Lauritzen (Eds.), The nature of statistical learning theory [Internet], 8, Springer, 1995, p. 188. http://portal.acm.org/citation. cfm?id=211359.

[63] Suykens J.A.K., Vandewalle J. Least squares support vector machine classifiers. Neural Process Lett. 9(3):293-300.

[64] N. Pombo, N. Garcia, V. Felizardo, K. Bousson, Big data reduction using RBFNN a predictive model for ECG waveform for eHealth platform integration, in: e-health networking, applications and services (Healthcom), 2014 IEEE 16th international conference on, 2014, pp. 66-70.

[65] C. Chen, C. Yan, Y. Li, A robust weighted least squares support vector regression based on least trimmed squares, Neurocomputing 168 (2015) 941-946.

[66] B.G. Horne, C.L. Giles, An experimental comparison of recurrent neural networks, in: G Tesauro, DS Touretzky, TK Leen (Eds.), Advances in neural information processing systems 7 [Internet], MIT Press, 1995, pp. 697-704. http://papers.nips.cc/paper/1009-an-experimental-comparison-of-recurrentneural-networks.pdf.

[67] F.E. Harrell, K.L. Lee, R.M. Califf, D.B. Pryor, R.A. Rosati, Regression modelling strategies for improved prognostic prediction, Statis Med 3 (2) (1984) 143-152.

[68] N.R. Draper, H. Smith, E. Pownell, Applied regression analysis, 3, Wiley, New York, 1966.

[69] S.A. Dudani, The distance-weighted k-nearest-neighbor rule, IEEE Trans Syst Man Cybern SMC-6 (April(4)) (1976) 325-327.

[70] L.A. Zadeh, Fuzzy sets, Inf Control 8 (3) (1965) 338-353.

[71] T. Kohonen, Improved versions of learning vector quantization, in: Neural networks, 1990, 1990 IJCNN international joint conference on, 1, 1990, pp. 545-550.

[72] L.E. Baum, T. Petrie, Statistical inference for probabilistic functions of finite state markov chains, Ann Math Statis 37 (6) (1966) 1554-1563.

[73] Watkins A., Timmis J., Boggess L. Artificial immune recognition system (AIRS): an immune-inspired supervised learning algorithm. Genetic Program Evol Mach. 5(3):291-317.

[74] P.N. Belhumeur, J.P. Hespanha, D.J. Kriegman, Eigenfaces vs. Fisherfaces: recognition using class specific linear projection, IEEE Trans Pattern Anal Mach Intell 19 (July(7)) (1997) 711-720.

[75] Z. Pawlak, Rough set theory and its applications, Inf Syst J 29 (7) (1998) 7-10.

[76] N. Pombo, P. Araújo, J. Viana, Knowledge discovery in clinical decision support systems for pain management: a systematic review, Artif Intell Med 60 (1) (2014) 1-11.

[77] C.M. Bishop, Pattern recognition and machine learning (Information science and statistics), Springer-Verlag New York, Inc, Secaucus, NJ, USA, 2006.

[78] Breiman L. Bagging predictors. Mach Learn. 24(2):123-140.

[79] D. Casasent, Y.-C. Wang, Automatic target recognition using new support vector machine, in: Neural networks, 2005 IJCNN '05 proceedings 2005 IEEE international joint conference on, 1, 2005, pp. 84-89. 
[80] D. Ruiz-Fernández, A.M. Torra, A. Soriano-Payá, O. Marín-Alonso, E.T. Palencia, Aid decision algorithms to estimate the risk in congenital heart surgery, Comput Methods Program Biomed 126 (2016) 118-127.

[81] L. Lin, C. Jin, Z. Fu, B. Zhang, G. Bin, S. Wu, Predicting healthy older adult's brain age based on structural connectivity networks using artificial neural networks, Comput Methods Program Biomed 125 (2016) 8-17.

[82] C. Colak, E. Karaman, M.G. Turtay, Application of knowledge discovery process on the prediction of stroke, Comput Methods Program Biomed 119 (3) (2015) 181-185.

[83] A.R. Hassan, M.A. Haque, Computer-aided obstructive sleep apnea identification using statistical features in the EMD domain and extreme learning machine, Biomed Phys Eng Express 2 (3) (2016) 035003.

[84] A.R. Hassan, M.I.H. Bhuiyan, A decision support system for automatic sleep staging from $\{E E G\}$ signals using tunable Q-factor wavelet transform and spectral features, J Neurosci Methods 271 (2016) 107-118.

[85] A.R. Hassan, M.I.H. Bhuiyan, Automatic sleep scoring using statistical features in the \{EMD\} domain and ensemble methods, Biocybern Biomed Eng 36 (1) (2016) 248-255.

[86] A.R. Hassan, M.I.H. Bhuiyan, Computer-aided sleep staging using complete ensemble empirical mode decomposition with adaptive noise and bootstrap aggregating, Biomed Signal Process Control 24 (2016) 1-10.
[87] A.R. Hassan, M.A. Haque, Computer-aided sleep apnea diagnosis from single-lead electrocardiogram using dual tree complex wavelet transform and spectral features, in: 2015 international conference on electrical electronic engineering (ICEEE), 2015, pp. 49-52.

[88] A.R. Hassan, Automatic screening of obstructive sleep apnea from single-lead electrocardiogram, in: Electrical engineering and information communication technology (ICEEICT), 2015 international conference on, 2015, pp. 1-6.

[89] T. Wang, J. Qiu, H. Gao, Adaptive neural control of stochastic nonlinear time-delay systems with multiple constraints, IEEE Trans Syst Man Cybern Syst (99) (2016) 1-9.

[90] J. Qiu, H. Gao, S.X. Ding, Recent advances on fuzzy-model-based nonlinear networked control systems: a survey, IEEE Trans Ind Electron 63 (February(2)) (2016) 1207-1217.

[91] T. Wang, J. Qiu, S. Yin, H. Gao, J. Fan, T. Chai, Performance-based adaptive fuzzy tracking control for networked industrial processes, IEEE Trans Cybern 46 (August(8)) (2016) 1760-1770.

[92] T. Wang, H. Gao, J. Qiu, A combined fault-tolerant and predictive control fo network-based industrial processes, IEEE Trans Ind Electron 63 (August(4)) (2016) 2529-2536. 